\title{
From fundamental quantum optics to quantum information technology: the personal journey of Sir Peter Knight
}

Xiaosong Ma

Nanjing University, School of Physics, Nanjing, China

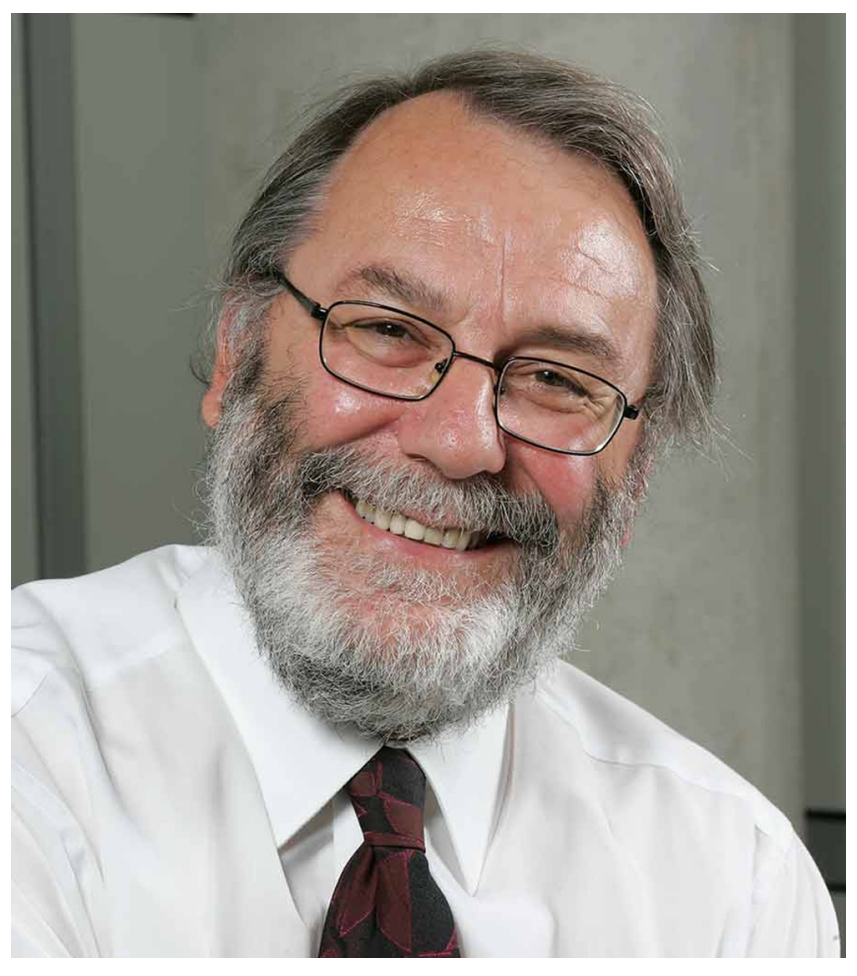

Professor Sir Peter Knight, Imperial College London, UK

Xiaosong Ma: What inspired you to choose quantum optics as your major when you were a student, and can you share your experience or research journey in this field?

Peter Knight: Quantum optics, as a discipline, was more or less formed during the time I've been active in the area. I started thinking about things that we would now call quantum optics in the middle of the 1960 s when I was a student. As a subject, it was really hardly developed at that point, but looking in particular at the way that the quantum nature of light would manifest itself in regular laser type experiments was then beginning to emerge. The field initially was called "quantum electronics" at that point, and "quantum optics" was a term used by very few people who started to worry about what the quantum nature of light would do.

I started in this area as an undergraduate. Like many students, to demonstrate that you can do something original of your own, I had to do a project. The project I chose was to work on optical pumping: making a cesium cell and looking really carefully at ways in which you could monitor coherent transients in optical pumping. In particular, I was measuring the way that Rabi oscillations could be monitored and looking at decoherence-which sounds like the things we worry about

(C) The Authors. Published by SPIE and CLP under a Creative Commons Attribution 4.0 International License. Distribution or reproduction of this work in whole or in part requires full attribution of the original publication, including its DOI. [DOI: 10.1117/1 .AP.3.6.060501] now, but this of course was in the radio frequency regime. This was experimental activity that was done with really simple experiments and simple apparatus. Of course, this is before tunable lasers. This experiment was all done with thermal light sources.

Firstly, I found the field really fascinating. And secondly, I was probably deluded in thinking that I could be an experimentalist, because the apparatus was really simple. So, when I started my $\mathrm{PhD}$, my project was to do a kind of mix of theory and experiment, and it very quickly emerged that I was totally incompetent, really useless as an experimentalist. And I think the people in the lab were really delighted when I said, "I think it would be best if I turned pretty much to theory." So my $\mathrm{PhD}$ was in theory. But I've always maintained a really close engagement with experimentalists around me. Rather than being a theorist only in a theoretical environment, I have always enjoyed working with colleagues who were doing experiments so we could feed off each other. So that journey was a kind of accident, but it was fascinating and it's something that I've done ever since, so in all of my roles and positions, I've always had people I could talk with who were doing wonderful experiments. I guess that's kind of unusual in many places in the UK, where theoreticians and experimentalists are often in separate departments.

I did my PhD in that area, and then I went off to the United States as a postdoc, working with Joseph Eberly in the United States. And again, you know, a really powerful theoretician but always working with experimentalists. I had a wonderful three years in Eberly's group as a postdoc, working with really great people and really understanding, for the first time, how we could put together a group, how to plan a long-term career.

I came back to the UK in 1974. Around that time, the number of people interested in quantum optics theory in the UK with proper academic jobs was probably about four or five people, in the whole country. It was an extreme minority interest. But it was something that was becoming really exciting. Coming in at the very beginning of a subject area was always a wonderful experience. When I came back to the UK, one of the leaders in the field was Rodney Loudon at the University of Essex. Of those three or four people other than me working in quantum optics, he was someone who was really influential in my career. And again, Rodney had worked very closely with experimentalists.

I had various fellowships, and that gave me my chance to have my first graduate students of my own. I basically co-supervised graduate students in the US. In particular, some of Eberly's students worked primarily with me. Peter Milonni, for example, worked primarily with me. That already gave me the experience of working with really talented people to hit tough problems. Working in isolation, on your own, you could do something; but working with a group of like-minded people, you could do so much more.

In 1979 I moved to Imperial College, and I've basically been at Imperial College ever since. My group expanded, became almost a subdepartment of the department, always with many experimental colleagues involved. And my experience of working in the US and then coming back to the UK really demonstrated to me that this kind of activity was an international endeavor. I really benefited from collaborating with people from around the world, so my group became extremely 
international during its most productive time. I do believe that's something that really is a lesson for everybody: working with the best around the world, you can do so much.

Xiaosong Ma: That's fantastic. You mentioned that it's good to be in the beginning of a subject, such as quantum optics in 1970s. To follow up on that, what do you think about, for example, quantum information, which is also an emerging technology nowadays. Which phase do you think we are in now for this technology?

Peter Knight: That's a really good question. Quantum information science was really born out of quantum optics, where there was a lot of basic research done over a very long period. Quantum computing, quantum cryptography, and so on, all came out of really basic research. The implementation to make these things practical has taken quite a while. I think we are pretty close to understanding a lot of the physical phenomena. While rolling this out as technology is a real challenge, we do understand many things. In some areas, we're making tremendous progress, yet underneath it there are still some puzzles that need to be worked out.

One of the questions you were going to ask me and I'm going to jump ahead to it was, what would you do if you were starting out as a student now? There's a tremendous amount of interest all around the world in quantum computing. People are making basic prototypes that are working wonderfully well, and we saw this huge advance in China recently using a superconducting chip, which could demonstrate the scaling as you increase the number of qubits. There was also the squeezed state boson sampling done in China as well. They already begin to demonstrate the idea that you can get a quantum advantage.

Now, maybe I'm alone in worrying about this, but I don't think we really understand the origin of the quantum advantage. Why do we get a speed up? Where's it coming from? We know that there's quantum entanglement, we know there's coherence, we know that there's quite a lot of worry about nonlocality. But, the engine of quantum computing is poorly understood. What is it that gives us the advantage? So, if I were starting out all over again, I would say that's one of the really big problems to address: we know that it's beginning to work, but why does it work? And by the way, I very rarely say that sentence to politicians who are funding us.

Xiaosong Ma: We will make sure this will not appear in the interview transcript.

Peter Knight: No, you should keep it in. Because, we have an unknown power. We know it's got power and we know it's going to give us tremendous advantage in information technology, but the origins of the advantage are still to be explored. Now, coming back to the beginning of your question earlier, you talked about starting out in the field. If you have a really established subject and you are a young scientist, it's quite hard to make your mark in the subject, because there's so much already done. This is a feeling often with young students in established fields, that they can only do a little bit of incremental work. But in a new subject, you can be a game changer-you can provide completely new ways of looking at things, which are revolutionary. Now I'm going to give you some examples of people that I worked with in the past. One of my most famous former students was Artur Ekert. He came up with entanglement-based quantum cryptography in the final few months of his $\mathrm{PhD}$. As it happens, we'd been interested in quantum correlations for a long time. He had done a great deal of work on Bell inequalities, so it was a natural thing for him to investigate towards the end of his $\mathrm{PhD}$. So, there you have an example: quite a new researcher in the field can transform the subject. Another example of someone who was not my student, but someone I know quite well is Andrew Steane, in Oxford. He was the co-inventor of quantum error correction as an early career researcher. You can see how young people in an emerging field could be transformative. That's why, in quantum information science, we're getting some of the brightest young people in the world wanting to work on it. It's fascinating and there're huge challenges, but they know they can make a difference personally.

Xiaosong Ma: That's amazing. You've just mentioned Artur Ekert, his research on Bell's inequality, correlations, etc., primarily on the foundations of quantum physics. That's very fundamental research, I would say. Do you envision that this fundamental research could be transformed into application, such as entanglement-based QKD at the very beginning?

Peter Knight: I think some of the advances we're seeing still require quite a lot of engineering investigation. We can see that we can build entanglement-based quantum communications. After all, we can now do long-distance quantum communication along optical fibers and in free space, and even via satellite as the Micius experiment demonstrated. So there is tremendous potential for it. But you know the moving around of information security is only a part of the issue. You've got to be able to work on the entire security of the system, how is it implemented. So we need security proofs of the real apparatus, not the idealized apparatus. And that's still work in progress, because you really wouldn't want to reengineer the entire communications system and find that through the way that the engineering is implemented it becomes faulty.

I think a lot of work is underway on implementation protocols and security of these things. But it is wonderful to see that something as strange as nonlocal Bell correlation is generating a new industry. I did meet John Bell a number of times and I think he would have been astonished by all this. Bell was actually quite a practical person. For most of his career, he was an accelerator scientist, working on designs of accelerators - that was what he was paid for. The fundamental work was a kind of sideline hobby of his. I don't think he would have ever thought that his own work would have been the foundation of a whole new industry, which it is.

We knew that quantum could be transformative. Not just the entanglement-based side of things, but coherence. After all, atomic coherence is a driver of one of the main features of modern technology: it's what under underlies GPS, atomic clocks in satellites, and so on. So, our navigation and timing systems all around the world are wholly dependent on the preservation of coherence amongst atomic spins. People don't realize that. When people say quantum technology is a new thing yet to prove itself, it's always worth reminding them that we've come a long way: GPS contributes enormously to the world economy, and it depends on quantum superpositions.

Xiaosong Ma: We have talked about superposition, coherence, and also entanglement. Those are main features for quantum systems, and they are applied to this new quantum revolution. Do you think there are still undiscovered quantum features that could be potentially useful?

Peter Knight: In terms of usefulness, I think we're beginning to understand where the limitations are, and what the advantages are. In quantum sensors that can detect, for example, electromagnetic fields, or gravitational irregularities, we're already seeing that. And again, it's worth remembering that these things move very fast. For example, I mentioned that when I first started, I was worrying about optical pumping, way back in 1967. It's now possible to build really small optical pumping cells that can detect the electromagnetic signals in the brain. Of course, all of this is used a lot in brain scanners, huge superconducting coils, and so on. But quantum technology is working at ways in which we can now build a really compact sensor that's like a cycle helmet and will monitor brain activity. One of the developments in 
that area is that some of our colleagues in quantum technology in Nottingham have built a brain sensor of that kind that's already being used by surgeons looking at epilepsy, so it's being used in hospitals. So, this is a quantum sensor of brain activity that's being used to direct a surgeon's knife. That's amazing, really amazing.

If you work in a built environment, something that's been there for a long time: one of the dangers of any new construction development is what's under your feet-where are the tunnels, where are the voids. And in big cities where there's a lot of redevelopment going on, it's really difficult to work out what's under your feet. We waste an awful lot of money in civil engineering on doing that. A quantum interferometer that can measure gravitational differences can already tell you what's under your feet. There are a number of people around the world who are building basically cold-atom interferometers that measure the ground infrastructure. Firstly, it's fascinating - you can map the underworld-but also there's the practical advantage of not wasting billions digging up the road trying to work out where the pipe work is. These are interesting things because the way the interferometer works is looking at really fundamental aspects of quantum physics. And we're applying it to work out where the drain is - a wonderful combination of the practical and the unknown!

Now we are still thinking about some of the more fundamental things. I mentioned that we don't really know what the power quantum computation derives from, but equally, most of what we're doing in this subject is built on an assumption that our current ideas of quantum mechanics will remain true-in other words, superpositions and linearity. Well, is it true? Or are we going to have evidence from really sensitive experiments that would demonstrate that there's something more out there than what we see already from linear quantum mechanical superpositions? People are beginning to think about that, because we know that gravity changes things. So, there are some really fundamental things to do in the area. And again, around the world, people are starting to look at ways in which this extraordinary sensitivity of quantum technology could be used to detect whether we really do understand all the fundamental laws of nature. Obviously, we promise our taxpayers we're going to be useful. But equally, the sensitivity of the experimentation can enable us to explore new things in fundamental science.

Xiaosong Ma: That's really intriguing. To use quantum mechanics to explore gravitational effects would be very interesting for the fundamental research. I guess you meant that can also be a potential new area for researchers, to use sensitive quantum sensors to explore gravitational effects.

Peter Knight: People are starting to look at whether quantum technology could be a useful vehicle to detect dark matter. Dark matter is one of the things that is a huge failure of modern physics. Most of the universe is made up of a stuff that we don't know about: dark matter, dark energy. We don't know what it is! What can we do in terms of sensitive measurements that will tell us a bit about this strange majority component of the universe? Quantum technology is already beginning to offer clues about how we would do this. The interferometer that could detect the gravitational changes in your built environment-if you can make that work at scale, you can start to detect dark matter, perhaps. That's something already occupying some really clever people around the world.

Xiaosong Ma: To follow up, you've mentioned that we already harness quantum features by using GPS, and also we are starting to use sensors for biomedical applications, as well as metrology tasks. How do you foresee this technology will further change our daily life?

Peter Knight: I've given a lot of thought to this question about how it influences, for example, our neighbors, the people who pay our salaries. One of the obvious things to say is that we can be assured that the internet is resilient and robust through encryption techniques that wholly rest on something being difficult—not impossible, but difficult. Obviously, factoring is what I'm thinking about. But security based on difficulty is only secure if it remains difficult. A classical computer would find tasks like factoring extremely difficult, but a quantum computer can do it so much more easily. That's part of the threat to business. What we must do is we must assume that in about 10 years, pretty much around 10 years everybody agrees, it's quite likely that we will have a large-scale quantum computer somewhere. As soon as you have a large-scale quantum computer, all of our encryption techniques that we use-RSA, the whole basis of HTTPS-become vulnerable. And that means that you no longer have a trusted internet ecosystem. The big change that will affect everybody is that we'll have to roll out quantum-safe encryption in order to do transactions, to pay our bills, to do our banking, and so on. That will affect everybody. So, quantum-safe approaches - they might be classical, a new source of algorithms, they could depend on quantum key distribution or whatever - they will affect everybody. If we're paying for stuff on the internet, we rely on the little padlock that appears on the website we're looking at to say yes, it's encrypted and secure. In ten years, we won't be able to assume that it's secure, because a quantum computer will make it insecure. That's where it will change everybody's life, and there're probably lots of other things.

Some of the applications for quantum technology I believe are yet to be discovered, because it's a new field and things happen that will surprise us. I don't know if you know how Bell Labs justified the investment in transistors? All amplifiers before transistors were thermionic valve amplifiers-big, power hungry, hot, full of extraordinary voltages. When Shockley and others started to think about a semiconductor that could make a transistor, they got it accepted by the Bell Labs organization. They got them to agree to invest with one application in mind, the killer app that enabled them to invest: to build a hearing aid for the deaf. Because all they wanted was a compact amplifier, and that's what you need for a hearing aid. Stanley Williams at HP Labs in the United States always says that all we need in quantum technology is the quantum hearing aid. In other words, some small development which will enable people to confidently invest and build something. Because once you've done that, millions of applications emerge from everywhere. When they built a transistor, they had no idea of the way that they were going to change the world-except for the hearing aid. So, all we need, to quote Stan Williams, is a quantum hearing aid. It will work, and then people will say, hey I can use this in all these other applications as well. Surprises happen in new emerging technology, and I haven't even thought of the big things that are bound to happen. Be prepared for surprises.

I think it's really interesting where we see this investment race going on around the world. It seems as if governments have a kind of virility test where they think, oh we've got to invest because everybody else is investing. This is called fear of missing out. I think that we have to be all really careful not to hype up quantum tech, to over promise, to say it's going to be revolutionary in every aspect. We've really tried not to hype it up, but I think we are already seeing things that are transformative, so although we try to avoid hype, we are seeing some wonderful things.

Xiaosong Ma: Exactly. Speaking of investments in the quantum technology industry, as far as I know, you are one of the leaders in setting up the quantum technology program in the UK. Can you briefly tell us about the history? Was it difficult at the beginning? Where do you see this project in the next five years?

Peter Knight: We've had a history of quality scientific research in quantum optics and atomic physics and so on in the UK which was verified by external review and so on. But we could see, by around 
2010 roughly, that some of the challenges of actually understanding how to manipulate and control individual quantum systems-basically our focus then-could then be applied in terms of things that had engineering importance. Around that period, we started to think about how to move quantum science to quantum technology. It was an exciting period because in the UK we had real strengths in the area. But what we realized is that if we could bring the partners-industry, government departments, universities-together, we could do so much more if we coordinated. We had this vision of this national program where we could bring everybody together to play a part. We started to talk about the idea, and we managed to get a fair bit of support from people about how this as an experiment could succeed. At that particular time in the UK we had a science minister who I think really understood where clever people could make a difference. Although we talked about it for quite a while, suddenly it got momentum. I remember in 2012-2013, we had to write short briefing notes for politicians about what quantum physics could do. And by short I really mean short, so our Finance Minister wanted a 4-page summary; and then the Prime Minister said, could I have a 1-page summary? Then it all moved very, very fast. At the end of 2013 , we got a new commitment of $£ 270$ million - a lot of money in those days-of completely new money, to build this national program. To that we then added other things, so it grew in that first period to about half a billion. We built these hubs, we worked with industry, and so on. And then I helped to write the vision piece for another five years of funding, so we we've got about a billion now to do this. And that meant that we probably started in a coordinated program quite a long time before everybody else. Other countries always had very strong quantum science programs, but the idea that you could coordinate it was relatively new. It was tremendous fun doing this, bringing in people with completely different attitudesengineers, computer scientists, physicists, the government people who worry about the impact on policy, and so on. And it was interesting. Others began to note the way that we approach this, looking at quantum sensors, quantum communication, quantum imaging, and then quantum computing, and those four pillars have become the pillars of other people's programs around the world as well. So the European Union flagship mirrors this and so do the United States NQI programs as well, so I think we got it right, basically, but we got there a bit earlier.

Xiaosong Ma: My next question would be about your mentoring. I've noticed that many of today's leading physicists in the quantum optics field came from your group. Can you share with us your secrets, or your recipes for mentoring and advancing young scientists and students?

Peter Knight: I don't think there's a secret. I think it comes down to a number of human characteristics that I think are important. Firstly, respect for others-understanding that you may not have the secret of everything, therefore you should respect what other people are telling you. Respect, sympathy, and the ability to see that others deserve the chance that you had. I think in some areas of science, you see the big chief doing all the great stuff and getting all the credit, and a lot of people in subsidiary positions. That's one model of doing that kind of science. But in an emerging field, it may not be the best way to do it, because things come out in a surprising way. I really benefited from quite an early stage in my career from demonstrating independence. When I came back to start my own group, I think I was 27. Giving people an early opportunity to be independent is part of it, so when you've got really bright students don't regard them as a personal possession. They are not personal possessions, they are people who are changing the subject. I spent most of my life as a university teacher, and the whole point about a teacher is to foster new knowledge and to encourage the next generation to flourish - that's our job. So, getting the very best people in, giving them a chance to really excel, and also finding mechanisms by which they get early independence themselves. That's been the trick.

I'll run through some of my students, for example, and of course it will be hugely embarrassing for them to be so named, but I'm going to do it. I mentioned Artur Ekert and Artur got a fellowship immediately after his $\mathrm{PhD}$ and that gave him independence. Another really smart student is Stephen Barnett. Stephen Barnett was my student and again very quickly got fellowships. He's now a Royal Society research professor in Scotland. Let me continue with the students. Barry Sanders in Calgary was another student. Giving them a chance to demonstrate their individual strengths and powers is really important, because two things happen: they flourish and the field flourishes, which impacts us as well. So, I think mentoring is really important. I wish more people would play more attention to mentoring, because that's the way the field really effectively grows. Young people are not slaves. Young people change the world, and you mustn't put obstacles in front of them. Let me think of some of the other students like Vlatko Vedral, for example, now a professor in Oxford...many of them have gone on to do a really great job...Myungshik Kim, who is one of my successors at Imperial. And I can keep going on about these things. The trick on this one is respect, respect for what they can contribute, rather than their ability to follow orders from you. Because then the whole field benefits. Not only that, you'll still have good people wanting to work with you. Try not to be a tyrant, because then people get scared off.

Xiaosong Ma: Respect and giving students early independence are very important. My personal experience also benefits from that. I think this advice will be extremely helpful for young research group leaders, and all the others.

Peter Knight: You have a responsibility, therefore, when you're generating new sources of resource and funding, to make sure that funding can be used by the next generation as well, to create fellowship schemes and so on. Because that will enable this to happen. As well as pushing for the big programs on funding particular investigations, making sure that you've put resources in, in terms of skills, career development, and so on. And it works!

Xiaosong Ma: You have been in many important positions, such as deputy rector at the Imperial College, and president of the IOP, and president of the OSA, etc. I can imagine you must have a very busy schedule. How do you manage to keep research, administration, and life in balance?

Peter Knight: This is a really good question, and there is only one honest answer: you don't. You don't keep a balance. If you look at my career, when I was most productive in terms of research was when I had the least amount of external administrative responsibilities. My own personal scientific productivity really dipped when I started to take on the university leadership activity, because you can't really sensibly run a really high-level research program and run a university. If you look at my publication rate, you can see the Google Scholar graphs dip immediately after I started to do this. So, the honest answer is, you can just about keep research active as you manage these things and the key to a lot of this is finding the optimum balance, given your other responsibilities. When I was president of the OSA, that was a time when I didn't really have an enormous range of administrative responsibilities, so that was relatively straightforward, although it did mean getting on airplanes to the United States quite a lot.

Technically, I retired 10 years ago. When you work in my field, what does retirement mean? It means that they stop paying you, but you don't stop working. I live on my pension. That really means that I can strike out and try some new things that are high risk. Taking on a lot of what we're doing with the UK national program and so on 
is because I'm a free agent now. I've had a really great career, so I have the time to do it. I think that's another part of being convincing: when you're trying to persuade a government to put together a billion pounds to fund a program, if the person advocating it is not advocating it for personal gain but to propel the field, you get listened to. Whereas if I were still running an enormous research group, they would say, oh, Peter's just trying to get stuff for himself. You can't have a compelling vision that's trusted in that role. So, to be honest, retirement was a really good thing for me, because it meant that I could really propel these things along. But coming back to the basics of your question, you can't run a really world-class, high-level research activity and take on a lot of research responsibilities at the same time without terrible things happening. Productivity goes up and down, and so on, so you have to work out what is most beneficial. In the 1990s onwards, research time for me was easy and so on, but once you have a voice, you can use that voice to enable others to do things as well.

Xiaosong Ma: I believe the quantum researchers in UK should thank you for your advocacy to the government to provide stable funding over long periods of time. Moreover, such a national program also inspires other countries to invest in quantum technology. Therefore, I think all of us should be very grateful for your efforts.

Peter Knight: Thank you for saying that. Of course, it's the energy, enthusiasm, and interest in the entire community that make it worthwhile. When you see the things that are going around the world, it really is exciting in so many ways. I remember when I first arrived at the University of Rochester as a postdoc, I would go to lunch with the people in the offices around me. The people in the offices around me were Emil Wolf, Leonard Mandel, Joseph Eberly, and Carlos Stroud. If we had ever thought, any of us, that there would be a quantum technology industry, we'd have been very surprised. What can we learn from that? It takes time, basically, and persistence, to do this. But being a young researcher, being exposed to people like Mandel, Wolf, Eberly, and Stroud was just hugely important for me, because it demonstrated how the intellectual life of science can be so exciting that you want to be really a part of it. It consumes your life.

Xiaosong Ma: Yes, it's very exciting. I think you just mentioned the 1970s, around 1974, as a golden age for quantum optics in Rochester. You have so many colleagues whom nowadays we, as young researchers, can only access in the textbooks - but you have lunch with them every day...That's very exciting.

Peter Knight: I know. One of the great engines in photonics, for example, in quantum photonics, is called the Hong-Ou-Mandel dip: the amplitude for two photons interfere in an interesting way. And I can remember the first I heard about that was when Leonard Mandel said, "you know this works, if you put two identical photons into a beam splitter, they will come up that way or that way, but not that way and that way." And the rest of us that the lunch table said, "really?" When you hear things that you know are going to be just amazing, it's wonderful.
That's why you should do science- because it's part of the cultural experience of everybody: to get amazed by new developments. It's exciting.

Let me finish with one of my protégés, I can only claim him partly as a protégé because he wasn't my student, although he was a postdoc with me for a while, and then later became a faculty member and a professor. This is Terry Rudolph. Terry has done some of the most advanced thinking about fundamental science. He's one of the founders of a quantum computing company called PsiQuantum. PsiQuantum recently did a major series fundraising and was valued by NASDAQ just two weeks ago at $\$ 3.15$ billion dollars. Who would have thought that you could do that? He's still writing wonderful fundamental scientific papers, but he as the theoretician and others as well-Jeremy O'Brien leading the experimental side with Pete Shadbolt and others - have got this way of building a quantum engine which is now valued at $\$ 3$ billion dollars. Terry Rudolph, when he started on this thing, looking at fundamental physics, and then built what is basically one of the largest quantum computing companies in the world. That's an astonishing journey. But it's built on the foundations of decades of hard work from people around the world. I think this ability to work collectively together and get the very best around the world to work with us, is really intriguing. Terry is British, but of course he was brought up in Australia and Malawi, and Jeremy O'Brien is Australian. We look at this world talent that we've got, and it's been transformative. In China, you have Jianwei Pan, who is incredible in realizing large-scale quantum network and achieving quantum computation advantages. We've got leaders around the world who are changing us.

Xiaosong $\mathrm{Ma}$ is a professor in the School of Physics at Nanjing University. He obtained his BS degree from Nanjing University in 2003, his MS degree from Leiden University in 2005, and his PhD from University of Vienna in 2010. A subsequent postdoctoral research post in experimental quantum physics at University of Vienna, was followed by a postdoc in integrated quantum photonics at Yale University.

Peter Knight is a senior research investigator in the Physics Department at Imperial College, chair of the UK National Quantum Technology Programme Strategic Advisory Board, and chair of the National Physical Laboratory Quantum Metrology Institute. Prior to retiring in 2010 as a deputy rector (research), he was responsible for the Imperial research strategy. A former president of the Institute of Physics (2011-2013), he was a member of the Imperial College Management Board and Council, and a professor of quantum optics. He was knighted in the Queen's Birthday Honours List in 2005 for his work in optical physics. $\mathrm{He}$ was until 2008 principal of the Faculty of Natural Sciences at Imperial College London. He was head of the Physics Department, Imperial College London, from 2001 to 2005. A past president of the Optical Society of America, he was a director of the OSA Foundation. $\mathrm{He}$ is an editor of Contemporary Physics and serves on a number of other editorial boards. He is a Thomson-ISI "Highly Cited Author." After receiving his doctorate at Sussex University, he was a research associate in the Department of Physics and Astronomy at the University of Rochester and the Stanford University Physics Department and SLAC. He joined Imperial College in 1979 first as a lecturer, becoming a professor in 1988. 